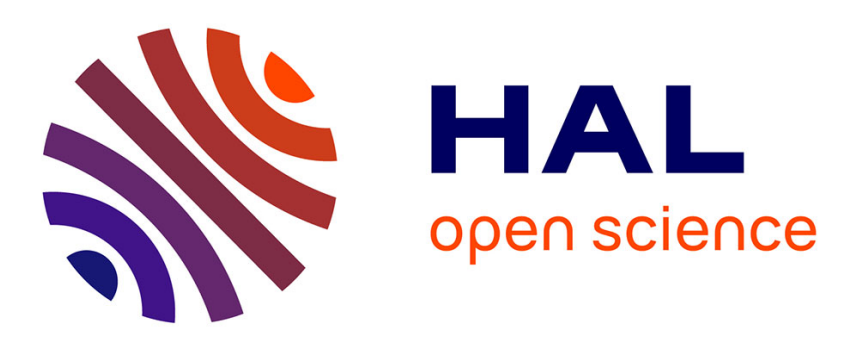

\title{
Une stèle funéraire d'Antioche au musée archéologique de Viuz-Faverges (Haute-Savoie)
}

Olivier Lempereur, Julien Aliquot

\section{To cite this version:}

Olivier Lempereur, Julien Aliquot. Une stèle funéraire d'Antioche au musée archéologique de Viuz-Faverges (Haute-Savoie). Syria. Archéologie, art et histoire, 2018, 95, pp.441-445. 10.4000/syria.7661 . halshs-02122257

\section{HAL Id: halshs-02122257 \\ https://shs.hal.science/halshs-02122257}

Submitted on 29 Jan 2020

HAL is a multi-disciplinary open access archive for the deposit and dissemination of scientific research documents, whether they are published or not. The documents may come from teaching and research institutions in France or abroad, or from public or private research centers.
L'archive ouverte pluridisciplinaire HAL, est destinée au dépôt et à la diffusion de documents scientifiques de niveau recherche, publiés ou non, émanant des établissements d'enseignement et de recherche français ou étrangers, des laboratoires publics ou privés. 


\title{
SYRIA ARCHÉOLOGIE, ART ET HISTOIRE
}

\author{
revue publiée par \\ l'Institut français du Proche-Orient
}

Tome 95

Année 2018

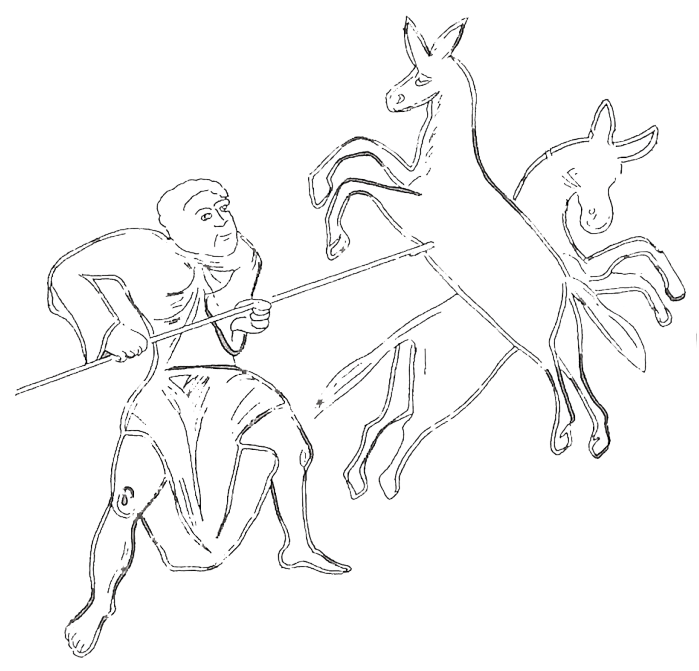

avec le concours du Ministère de l'Europe et des Affaires Étrangères (MEAÉ-DGM) et du Centre national de la recherche scientifique (UMIFRE 6, USR 3135)

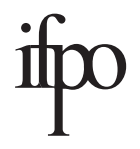

BEYROUTH 
Vignette de couverture : Détail d'une peinture murale de Qusayr 'Amra

(relevé C. Vibert-Guigue \& Gh. Bisheh, Les peintures de Qusayr 'Amra [BAH 179], Beyrouth 2007). 
Syria est une revue périodique annuelle publiée par l'Institut français du Proche-Orient

(UMIFRE 6, CNRS-MEAÉ, USR 3135).

\section{DIRECTION}

Éditeur :

Michel Mouton, directeur de l'Ifpo

Directeurs de la revue:

Françoise BRIQuel ChatonNEt, directrice de recherche au CNRS

Rédacteur en chef :

Dominique PIERI, directeur scientifique pour l'archéologie et l'histoire de l'Antiquité à l'Ifpo Françoise Briquel Chatonnet

\section{SECRÉTARIAT DE RÉDACTION}

Le secrétariat de rédaction de Syria, assuré par Gaëlle CoQUEUGNIOT (courriel : syria@cnrs.fr), est placé sous la responsabilité du rédacteur en chef de la revue. Les articles et communications scientifiques, les ouvrages dont on désire un compte rendu, et toute la correspondance afférente doivent êtres adressés à Gaëlle Coqueugniot, secrétariat de rédaction de Syria, Maison de l'Archéologie et de l'Ethnologie René-Ginouvès, 21, allée de l’Université, 92023 - Nanterre Cedex, France.

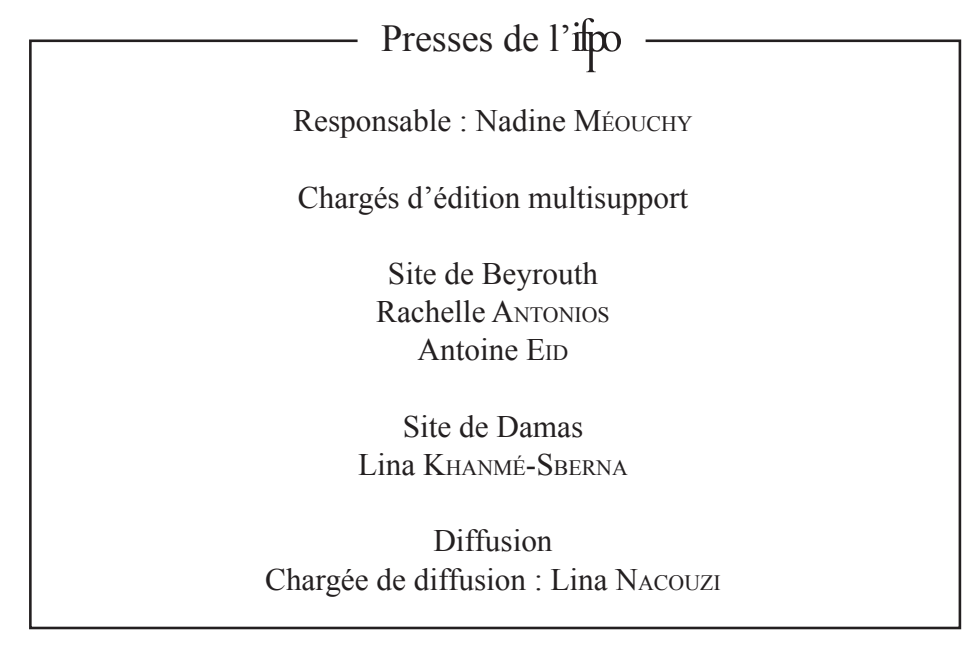

(C) 2018, Presses de l'Ifpo

B.P. 11-1424 Beyrouth, Liban

Tél./Fax : + 961 (0)1 420294

www.ifporient.org

Courriel : diffusion@ifporient.org

SYRIA en ligne :

http://journals.openedition.org/syria

http://www.persee.fr/web/revues/home/prescript/revue/syria

http://www.jstor.org/action/showPublication?journalCode=syria

ISSN 0039-7946

ISBN 978-2-35159-750-7

Dépôt légal : $2^{\circ}$ trimestre 2019 


\section{COMITÉ DE RÉDACTION}

Syria est administrée par un comité de rédaction incluant l'éditeur et les deux directeurs de la revue, entourés de :

Frédéric ALPI, ingénieur de recherche, CNRS, Ifpo, Beyrouth

Pascal Butterlin, professeur, Université Paris 1 Panthéon-Sorbonne

Denis Genequand, Université de Genève

Philippe QuenET, professeur, Université de Strasbourg

Carole Roche-Hawley, directeur de recherche, CNRS, Paris

Laurent THOLBECQ, professeur, Université libre de Bruxelles

François Villeneuve, professeur, Université Paris 1 Panthéon-Sorbonne

Jean-Baptiste Yon, directeur de recherche, CNRS, Lyon-Beyrouth

Ce comité officie également pour les ouvrages de la collection BAH (Bibliothèque archéologique et historique) de l'Ifpo.

\section{COMITÉ DE LECTURE}

\begin{tabular}{|c|c|c|c|c|c|}
\hline$F_{R A N C E}$ & ALLEMAGNE & ESPAGNE & JORDANIE & POLOGNE & RUSSIE \\
\hline R. BOUCHARLAT & H. KÜHNE & A. Almagro & Gh. BisheH & M. GAWLIKOWSKI & G. Koshelenko \\
\hline A. CAubet & W. ORthmanN & M. Molist & F. ZAYADINE & & \\
\hline D. ChARPIN & T. Ulbert & & & ROYAUME- $U_{N I}$ & SUISSE \\
\hline H. DE Contenson & T. WeBER & $\dot{E}_{T A T S}-U_{N I S}$ & LIBAN & M. MACDONALD & C. BonNet \\
\hline J.-M. DentZER & & G. W. Bowersock & L. BADRE & C. MANGO & R. StUCKу \\
\hline D. FeISSEL & BELGIQUE & D. PARDEE & H. SALAMÉ-SARKIS & E. Peltenburg & \\
\hline J.-B. HuMBERT & J. BALTY & & & & SYRIE \\
\hline J.-L. Нuот & J.-Ch. BALTY & ITALIE & & & M. AL-MAQDISSI \\
\hline J.-C. MARGUERON & F. de Callata & A. BARONI & & & S. MuHEISEN \\
\hline J.-P. SodINI & & A. INVERNIZZI & & & \\
\hline M. YoN & DANEMARK & P. MatThiAe & & & \\
\hline & J. LuND & S. RIBICHINI & & & \\
\hline
\end{tabular}

\section{RECOMMANDATIONS AUX AUTEURS/RECOMMENDATIONS TO AUTHORS}

Syria publie des articles en français, en anglais, en allemand, en italien, en espagnol.

Chaque article doit être accompagné d'un bref résumé d'une dizaine de lignes en français et en anglais (un résumé en arabe est ensuite réalisé et ajouté par l'éditeur). L'ensemble, article et notes, ne doit pas excéder 25 pages imprimées (sauf accord particulier préalable). Les articles reçus sont soumis sans délai à l'avis de deux rapporteurs désignés par le rédacteur en chef. Dès réception de leur rapport, les auteurs sont avisés de l'acceptation ou du refus de leur article, ou de demandes de modifications éventuelles.

D’une façon générale, la charte éditoriale, portant des recommandations plus complètes concernant la présentation des manuscrits, peut être téléchargée sur le site web de l'Ifpo/Presses de l'Ifpo : http://www.ifporient.org/node/87.

Tous les articles proposés pour publication dans Syria doivent être envoyés sous forme imprimée et numérique à Gaëlle Coqueugniot : syria@cnrs.fr, secrétaire de rédaction, et sous forme numérique uniquement à Françoise Briquel Chatonnet : francoise.briquel-chatonnet@cnrs.fr.

Syria publishes articles in French, English, German, Italian, Spanish.

Every submission should include a short abstract (10 lines) in French and English. An Arabic abstract is then written and added by the publisher. An article will not exceed 25 printed pages (including footnotes) unless agreed otherwise. Every article received is promptly submitted to two referees designated by the editor-in-chief. On receipt of their report, authors are informed whether their article has been accepted for publication or not, or are notified of any modification that may be required.

More generally, an editorial guide with more complete guidelines about the display of articles, can be downloaded from the website of Ifpo/ Presses de l'Ifpo/Charte éditorale: http://www.ifporient.org/node/87.

All articles submitted to Syria should be sent in hard copy and digital version to the assistant editor Gaëlle Coqueugniot: syria@cnrs.fr and in digital version only to Françoise Briquel Chatonnet: francoise.briquel-chatonnet@cnrs.fr. 


\section{SYRIA, TOME 95, ANNÉE 2018}

\section{SOMMAIRE}

ISSN 0039-7946

ISBN 978-2-35159-750-7

Éditorial

I - Dossier : Sur les routes de Syrie et d’Asie Mineure - Hommage à Maurice Sartre (coordonné par Catherine Grandjean)

Grandjean (C.), Avant-propos

BaLty (J.-C.), Cippes, autels funéraires et stèles militaires d'Apamée : typologie des monuments, modèles iconographiques et ateliers

Bowersock (G. W.), Religion in Early Fourth Century Roman Palestine: The Kfar 'Othnay Mosaics

GATIER (P.-L.), Le chameau et l'onagre, viandes des Ismaélites.

Mitchell (S.), L'alimentation en Asie Mineure à travers les siècles. Esquisse d'une histoire culturelle ....111

Aliquot (J.), Regio Parhalia. Genèse et administration d'un district domanial de la Syrie romaine

Boulay (T.), La liberté de Téos et le soutien d'Attale II à Alexandre Balas

Bader (N.) et Yon (J.-B.), Une inscription du théâtre de Bayt Ras/Capitolias 155

\section{II - Autres Articles}

Poupet (P.), Baldi (J. S.) et Harfouche (R.),

Matériaux pour céramiques à l'âge du Bronze, dans la vallée du Nahr Ibrahim (Liban)

AlachKar (S.), Euphrates Banded Wares et leur contexte funéraire. Un nouveau bilan

Blétry (S.), Duval (H.), Girardi (C.), Loupmon (T.) et Ruoob (A.),

Tell Keila, résultats de quatre années de recherches.

Mavrojannis (T.), Le commerce des esclaves syriens (143-88 av. J.-C.)

Kubiak-Schneider (A.), Une inscription palmyrénienne inédite à " Miséricordieux ».

Hutton (J. M.), Kumon (H.), McLaughlin (M.) et Atwood (P. L.), Two Palmyrene funerary

busts in the collection of the Nelson-Atkins Museum of Art, Kansas City, Mo

Kaizer (T.) et Raja (R.), Divine symbolism on the tesserae from Palmyra.

Considerations about the so-called "Symbol of Bel" or "Signe de la pluie".

BARAZE (M.), Un ensemble funéraire (IV - IX siècle) découvert dans le marché suburbain de Palmyre......317

Ahmad (T.), La fontana monumentale del santuario di Damasco - Siria (Sūq l-ṣàga).....

Claes (L.) et Tavernier (J.), Exit Gordianus, but how? Shapur's trilingual inscription revisited 
Gelin (M.), Les fortifications hellénistiques de Cyrrhus-Nebi Houri :

étude archéologique et géophysique.

Abdul Massin (J.), Développement et urbanisme du secteur méridional de Cyrrhus-Nebi Houri

Al-ShBiB (Sh.), La défense des villes à l'époque byzantine : alternance entre les tours et les bastions, tradition ou innovation?

$$
\text { III - VARIÉTÉS }
$$

Angevin (R.), Les industries lithiques de Tell Chuera et Kharab Sayyar (Syrie du Nord) :

nouvelles perspectives techno-économiques et implications historiques

Lempereur (O.) et Aliquot (J.), Une stèle funéraire d'Antioche au musée archéologique de Viuz-Faverges (Haute-Savoie)

SARTRE (M.), Retour vers la Coelé-Syrie

$$
\text { IV - NÉCROLOGIES }
$$

Ali Abou Assaf, la beauté morale des valeurs archéologiques

('Atil 23 septembre 1931-'Atil 1' mars 2018) [M. AL-MAQDISSI] 463

Javier Teixidor (13 novembre 1930-25 septembre 2017) [F. BRIQuel ChatonNET]

$$
\mathrm{V}-\mathrm{RECENSIONS}
$$

Les recensions sont désormais publiées exclusivement en ligne, sur le site https://journals.openedition.org/syria/

Les ouvrages suivants ont fait l'objet d'une recension dans l'année 2018 :

Nicolo Marchetti, Kinku, sigilli dell'eta del Bronzo dalla Regione di Gaziantep in Turchia (Orientlab 1), Bologne, Ante quem, 2011 [Pascal ButterLin]

Felix Höflmayer et Ricardo Eichmann (éd.), Egypt and the Southern Levant in the Early Bronze Age (OrientArchäologie 31), Rahden/Westf., Leidorf, 2014 [Christophe NiCOLLE]

Nicolò Marchetti (éd.), Karkemish. An Ancient Capital on the Euphrates, Bologne, Ante Quem, 2014 [Christine KePINSKI]

D. Praet et B. Bakhouche (éd.), Franz Cumont. Astrologie (Bibliotheca Cumontiana. Scripta Minora IV), Turnhout, 2014 [Jean LEMPIRE]

Mhamed Hassine Fantar, Maurice Sznycer, François Bron, Stèles à inscriptions néopuniques de Maktar, vol. 1 (Corpus des antiquités phéniciennes et puniques, France 3 / Tunisie 1, Mémoires de l'Académie des Inscriptions et Belles-Lettres 51), Paris, Diffusion De Boccard, 2015 [Jimmy DACCACHE]

Klaus Stefan Freyberger, Christine Ertel $\dagger$, Kathrin Tacke, Hassan Hatoum, Kanatha von hellenistischer bis spätantiker Zeit. Band I : Die Heiligtümer. Orte der Herrschaft und urbane Kommunikationszentren (Damaszener Forschungen 16), Mayence, Philipp von Zabern, 2015 [Maurice SARTRE] 
Karel J. H. Vriezen et Ute Wagner-Lux (éd.), Gadara-Umm Qès II. The Twin Churches on the RomanByzantine Terrace and the Excavations in the streets (Abhandlungen des Deutschen Palästina-Vereins 30/2), Wiesbaden, Harrassowitz, 2015 [Bertrand RIBA]

Zbigniew T. Fiema, Jaakko Frösén et Maija Holappa (éd.), Petra - The Mountain of Aaron. The Finnish Archaeological Project in Jordan. II. The Nabataean Sanctuary and the Byzantine Monastery, Helsinki, Societas Scientiarum Fennica, 2016 [Maurice SARTRE]

Emmanuel Laroche, Études anatoliennes (Subartu 37), Turnhout, Brepols, 2016 [Alice Mouton]

Dominique Parayre (dir.), Le fleuve rebelle, Géographie historique du moyen Oronte d'Ebla à l'époque médiévale, Actes du colloque international tenu les 13 et 14 décembre 2012 à Nanterre (MAE) et à Paris (INHA) (Syria Supplément IV), Beyrouth, Presses de l’Ifpo, 2016 [Jean-Louis Huot]

Hasan PeKer, Texts from Karkemish I. Luwian Hieroglyphic Inscriptions from the 2011-2015 Excavations (OrientLab Series Maior 1), Bologne, Ante quem, 2016 [Alice Mouton]

Philippe Quenet (dir.), Ana ziqquratim. Sur la piste de Babel, Strasbourg, Presses Universitaires de Strasbourg, 2016 [Cécile MicheL]

Shigeo Yamada et Daisuke Shibata (éd.), Cultures and Societies in the Middle Euphrates and Habur Areas in the Second Millennium BC-I. Scribal Education and Scribal Traditions (Studia Chaburensia vol. 5), Wiesbaden, Harrassowitz Verlag, 2016 [Bertrand LAFONT]

Cédric BRÉLAz (éd.), L'héritage grec des colonies romaines d'Orient. Interactions culturelles dans les provinces hellénophones de l'empire romain, Paris, De Boccard, 2017 [Maurice SARTRE]

Françoise Briquel Chatonnet et Muriel Debié, Le monde syriaque. Sur les routes d'un christianisme ignoré, Paris, Les Belles Lettres, 2017 [Sebastian BRock]

Ross Burns, Origins of the Colonnaded Streets in the Cities of the Roman East, Oxford/New York, Oxford University Press, 2017 [Catherine SALIOU]

Boris Chrubasik et Daniel KIng (éd.), Hellenism and the local communities of the Eastern Mediterranean, 400 BCE-250 CE, Oxford, Oxford University Press, 2017 [Maurice SARTRE]

Aaron Tugendhaft, Baal and the Politics of Poetry (The Ancient Word), New York, Routledge, 2018 [Stéphanie ANTHONIOZ] 



\title{
UNE STÈLE FUNÉRAIRE D'ANTIOCHE AU MUSÉE ARCHÉOLOGIQUE DE VIUZ-FAVERGES (HAUTE-SAVOIE) *
}

\author{
Olivier LEMPEREUR \\ Chercheur associé, UMR 5189 HiSoMA \\ olivier.lempereur@univ-savoie.fr \\ Julien ALIQUOT \\ CNRS, UMR 5189 HiSoMA \\ julien.aliquot@mom.fr
}

Né en 1927 à Alep d'un père militaire en poste dans cette ville de Syrie, Guy Gens passa sa jeunesse au Proche-Orient et s'installa à Beyrouth dans les années 1950. Il y enseigna l'histoire-géographie jusqu'en 1970. Son séjour libanais lui donna l'occasion de fréquenter les principaux sites archéologiques de la région et de constituer une collection d'antiquités principalement obtenues auprès de marchands beyrouthins. Établi en Haute-Savoie et continuant à enseigner à Chamonix, puis à Annecy, il visita à plusieurs reprises le musée de Viuz-Faverges, avant de léguer sa collection à l'association qui gère encore aujourd'hui cette institution. Son décès, survenu le 19 juin 2012, décida de l'entrée de ses acquisitions dans les collections du musée. La donation fut effective dès le 12 septembre de la même année.

Parmi le matériel lapidaire issu de ce legs, un petit monument inscrit en grec attire l'attention ${ }^{1}$. Affecté du numéro 119 dans la collection Guy Gens, il porte actuellement le numéro 437 dans l'inventaire du musée (fig. 1-2). Il s'agit d'une stèle funéraire parallélépipédique de marbre gris-blanc, haute de $37 \mathrm{~cm}$, large de $25,5 \mathrm{~cm}$ et épaisse de $8,5 \mathrm{~cm}$, conservée dans un bon état à l'exception d'une échancrure visible en bas. Contrairement au revers, qui reste grossièrement aplani au pic, l'avers du monument est soigneusement ravalé. Sa partie supérieure est ornée, à

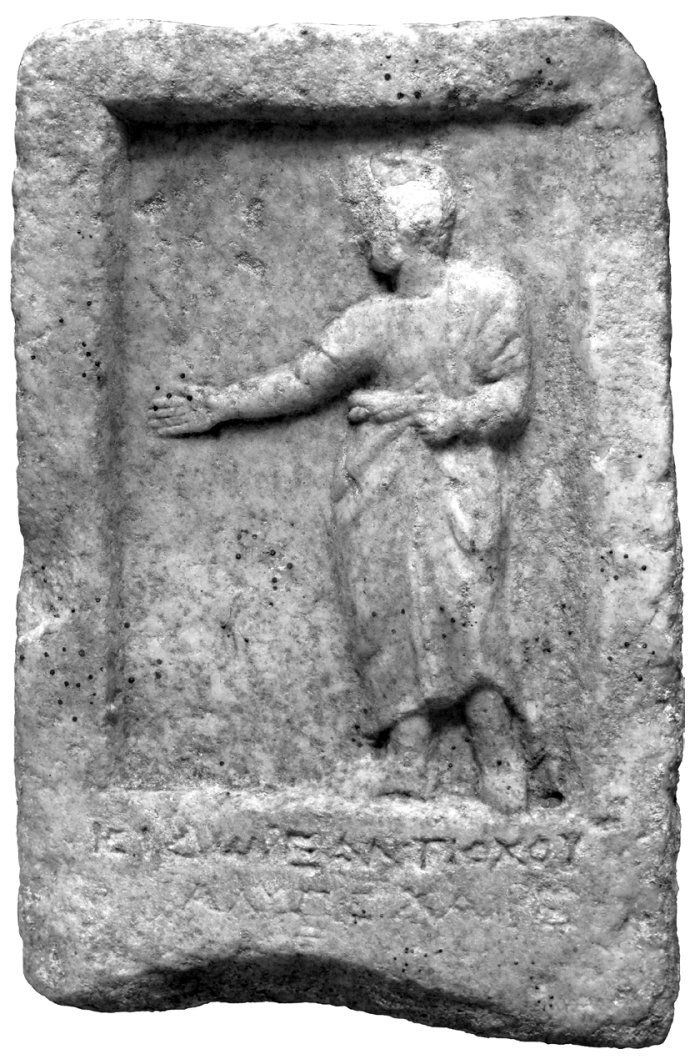

Figure 1. La stèle funéraire d'Antioche au musée archéologique de Viuz-Faverges, inv. 437 (c) Olivier Lempereur 2017.

* C'est avec plaisir que nous remercions Alain Piccamiglio qui, en nous accueillant au musée, nous a fourni de précieux de renseignements et laissé toute liberté d'étudier la collection Guy Gens. Notre reconnaissance va aussi à la direction de l'association des Amis de Viuz-Faverges, qui nous autorise à publier la stèle funéraire d'Antioche.

1. On trouvera en annexe la liste des autres objets de la collection Guy Gens légués au musée archéologique de ViuzFaverges. 
l'intérieur d'un cadre rectangulaire de $24,5 \mathrm{~cm}$ de haut sur $17 \mathrm{~cm}$ de large, d'un bas-relief qui représente un homme imberbe debout à gauche, vêtu d'une tunique à manches courtes et d'un long manteau noué à la taille. Le personnage porte la main gauche à la ceinture. Il tend le bras droit vers la gauche, dans un geste de salutation. La partie inférieure de la stèle contient une épitaphe de deux lignes, gravée en lettres régulières hautes de 1 à $1,3 \mathrm{~cm}$. La lecture du texte ne pose pas de problème.

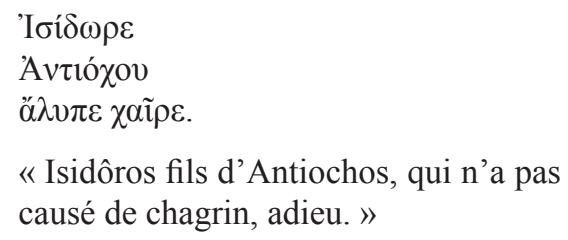

« Isidôros fils d'Antiochos, qui n'a pas causé de chagrin, adieu. »

Sous l'inscription, l'éclairage de la pierre en lumière rasante permet de lire les restes d'une autre épitaphe très effacée, dont seuls subsistent les deux mots de la formule finale, gravés sur la même ligne :

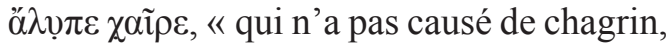
adieu $»$.

L'usage du marbre, la forme du monument, le style du bas-relief et le formulaire de l'épitaphe sont caractéristiques des stèles funéraires d'Antioche de Syrie (l'actuelle Antakya du Hatay turc), dont des centaines d'exemplaires sont dispersés dans les collections publiques et privées du monde entier ${ }^{2}$. Une partie de ces pierres tombales a transité par Beyrouth depuis le XIX ${ }^{\mathrm{e}}$ siècle. Le troisième tome des Inscriptions grecques et latines de la Syrie (IGLS), consacré à la ville d'Antioche et à ses environs, signale à l'occasion leur passage sur le marché des antiquités dans la capitale du Liban actuel ${ }^{3}$. À Beyrouth, le Musée national conserve cinq monuments appartenant à la même série ${ }^{4}$. En France, le musée du Louvre en possède une quinzaine ${ }^{5}$. L'origine de deux d'entre eux, le premier au nom de Doulos (Ma 2470) ${ }^{6}$, le second au nom d'Hermas (Ma 2844) ${ }^{7}$, n’a pas été reconnue jusqu'à présent. La provenance beyrouthine encore affichée à leur propos correspond en réalité au lieu où ces objets ont été vus ou acquis.

La stèle du musée de Viuz-Faverges présente quelques aspects originaux. La représentation du défunt seul dans cette attitude de salutation n'est pas très courante à Antioche, mais il existe au moins un parallèle sur un monument déjà publié dans les $I G L S$ et conservé au musée du Hatay à Antakya ${ }^{8}$. Tout comme la formule finale, fréquente au Proche-Orient, en particulier en Syrie du Nord et en Phénicie ${ }^{9}$,

2. Sur ce point, le recueil épigraphique ancien de JALABERT \& MOUTERDE 1950 (=IGLS 3/1), p. 492-525, peut être complété par des travaux récents beaucoup mieux illustrés : Kondoleon 2000 ; Lafli \& Meischner 2008; Lafli \& Christof 2014. Pour la mise à jour du corpus des épitaphes, voir les publications recensées dans le Bull. ép. 2005, 497, 520 ; 2006,445 ; 2010, 586 ; 2015, $695 ; 2016,519$.

3. IGLS 3/1, 887, 908, 915, 924, 932, 948, 963, $970 ; 3 / 2,1114$.

4. Yon \& Aliquot 2016, p. 201-203, $\mathrm{n}^{\text {os }} 375-379$.

5. Ma 2470, 2843-2844, 4277, 4287, 4487, 4493-4494, 4496-4497, 4502, 5458-5459; AO 11245-11246. Ces stèles seront toutes republiées dans le catalogue des inscriptions grecques et latines du Proche-Orient au musée du Louvre, que J. Aliquot, P.-L. Gatier et J.-B. Yon préparent actuellement.

6. SAulcy 1853, pl. 4, fac-similé d'A. Thiollet; Waddington 1870, p. 442, nº 1852 ; Froehner 1865, p. 273 , no 186.

7. Saulcy 1853, pl. 4, fac-similé d'A. Thiollet; Waddington 1870, p. 442, nº 1851 ; Froehner 1865, p. 275, no 191. Seul Jean Marcillet-Jaubert, dans Villa, Rousseau, Heilbrun \& CAubet 1982, p. 228-229, $\mathrm{n}^{\circ}$ 271, indique « acquis à Beyrouth » (p. 228), avant de rapprocher la stèle des monuments funéraires d'Antioche. La légende de la figure 234 de BEL 2012, p. 334, reste ambiguë : « Berytos? (Beyrouth, Liban)».

8. IGLS 3/1, 938. Voir LAFLI \& Christof 2014, p. 166, n 13, avec la photo p. 178, fig. 13 (avec une datation hellénistique peu vraisemblable).

9. Sur la formule $\alpha \ddot{\lambda} v \pi \varepsilon \chi \alpha i \tilde{\rho} \varepsilon$, voir Yon 2003. 
l'onomastique grecque de l'épitaphe est assez ordinaire. Le défunt porte un nom théophore d'Isis, 'I $\sigma i \delta \omega \rho o \varsigma^{10}$. Cet anthroponyme est banal, mais son attestation à Antioche soulève à nouveau la question de l'origine éventuellement syrienne des Antiochéens répondant à ce nom et dont les sources littéraires et épigraphiques révèlent l'existence ${ }^{11}$, à Rhodes et à Palaipaphos, à l'époque hellénistique ${ }^{12}$, à Athènes, à l'époque romaine ${ }^{13}$, et peut-être à Rome au $\mathrm{I}^{\mathrm{er}}$ siècle après J.-C., selon le témoignage du médecin grec

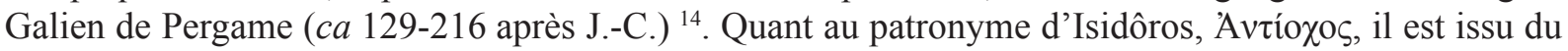
stock onomastique de la dynastie séleucide, sans surprise dans l'ancienne capitale des souverains grécomacédoniens de la Syrie hellénistique.

D'après l'écriture, l'épitaphe d'Isidôros date de la fin du $\mathrm{I}^{\mathrm{er}}$ siècle avant J.-C. ou du $\mathrm{I}^{\mathrm{er}}$ siècle après J.-C., au plus tard du début du II ${ }^{\mathrm{e}}$ siècle. La présence d'une autre inscription effacée en dessous du texte montre par ailleurs que la pierre était à l'origine destinée à un autre personnage ${ }^{15}$. La stèle du musée de Viuz-Faverges donne ainsi un exemple de plus du remploi des marqueurs funéraires dans les nécropoles d'Antioche sous l'Empire romain.

\section{Annexe : la collection Guy Gens au musée archéologique de ViuZ-Faverges}

Outre la stèle inscrite publiée ci-dessus, Guy Gens a légué 286 objets au musée archéologique de Viuz-Faverges. En voici la liste :

- 3 éléments d'architecture (chapiteau d'époque protobyzantine en calcaire, fragment de chapiteau corinthien, fragment d'architrave) ;

- 14 objets en pierre (massues ou casse-têtes, fusaïoles) ;

- 15 terres cuites (pesons, statuettes entières ou fragmentaires, dont deux syro-hittites datées de 1800-1600 av. J.-C.) ;

- 42 céramiques, la plupart complètes (lécythes, autres vases à parfums, aryballe, balsamaires, tasses, cruches, pots à anse, pichets/œnochoés, assiettes, verseuse, lagène, skyphos, petite marmite, lampes à huile) ;

- 2 amphores timbrées de type A-GRE Rho4 (productions de Rhodes ou apparentées);

- 113 monnaies ptolémaïques, séleucides, phéniciennes, provinciales romaines, dont plusieurs frappées à Antioche, auxquelles s'ajoutent plusieurs dizaines de monnaies romaines des $\mathrm{III}^{\mathrm{e}}$ $\mathrm{IV}^{\mathrm{e}}$ siècle, majoritairement émises en Orient, ainsi que quelques pièces byzantines et médiévales ;

- 2 objets en or (perle, plaque);

10. FeISSEL 1985, p. 97-98 et n. 66, réunit d'assez nombreux témoignages de l'onomastique isiaque à Antioche, auxquels on

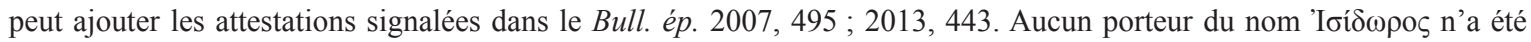
répertorié à Antioche jusqu'ici, mais on peut alléguer l'inscription d'une stèle funéraire de provenance probablement antiochéenne conservée au Middle Eastern Cultural Center à Tokyo (inv. 120). Sur les cultes isiaques dans la capitale syrienne, $c f$. en dernier lieu AliQuot 2014, p. 136-141.

11. Sur les questions que soulève l'identification des différentes cités de Syrie et d'Asie nommées Antioche dans la documentation épigraphique, voir notamment ROBERT 1973, p. 444-466 (= ROBERT 2007, p. 480-499), en particulier p. 444445 (= p. 480), pour les Antiochéens de Syrie.

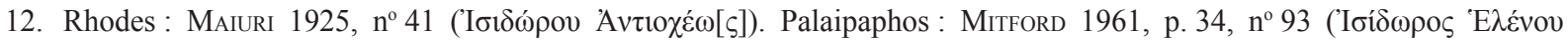

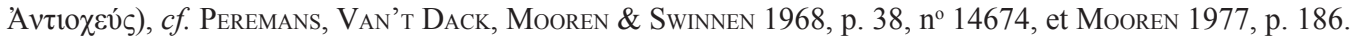

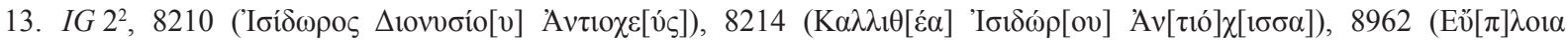

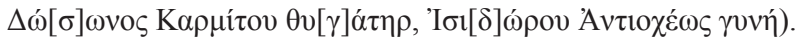

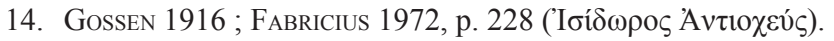

15. De même, Lafli \& Christof 2014, p. 166, n 13, notent eux aussi la trace d'une première inscription sous l'épitaphe $I G L S 3 / 1,938$. Voir aussi p. $164, \mathrm{n}^{\circ} 5$. 
- 41 objets en bronze : simpulum, encensoirs, pointes de lance et de flèche, poignards, épingles, pendentifs, miroir, instruments de chirurgie (spatules, sonde, petite cuillère, scalpel), anse de vase, bracelet, fibule, lampes ou brûle-parfums, plaques décoratives, boucles de ceinture, plateau de balance, bagues-clefs, fusaïole (?) ;

- 4 balles de fronde en plomb ;

- 28 objets en verre : bracelet, couvercles, bague, gobelet, balsamaires, amphorisque, flacons, entonnoir, tesselle, petit lion ;

- 2 colliers de perles ;

- 3 coquillages ;

- 16 lames de silex ainsi qu'un rognon.

\section{BibliograPhIE}

Aliquot (J.)

2014

BEL (N.)

2012

Aabricius (C.)

1972 Galens Exzerpte aus älteren Pharmakologen, Berlin.

FeIssel (D.)

1985 «Deux listes de quartiers d'Antioche astreints au creusement d'un canal (73-74 après J.-C.) », Syria 62, p. 77-103.

Froehner (W.)

1865 Musée impérial du Louvre. Les inscriptions grecques, Paris.

Gossen (H.)

1916 «Isidoros $29 »$, Realencyclopädie der klassischen Altertumswissenschaft 9/2, col. 2080.

JALABERT (L.) \& MOUTERdE (R.)

1950 Inscriptions grecques et latines de la Syrie, 3/1, Région de l'Amanus, Antioche, Nos 699-988 (BAH 46), Paris.
Kondoleon (C.)

2000 «Grave reliefs of Antioch », C. Kondoleon (éd.), Antioch, the lost ancient city, Princeton, p. 139-141.

LAfli (E.) \& Christof (E.)

2014 «New Hellenistic and Roman Grave Reliefs from Antioch », A. ÖZfIRAT \& Ç. UyGun (éd.), Uluslararast çağlar boyunca Hatay ve çevresi arkeolojisi sempozyumu bildirileri, 21-24 Mayis 2013 Antakya/ The Proceedings of the International Symposium on the Archaeology of Hatay and its Vicinity through the Ages, 21-24 May 2013 Antakya, Antakya, p. 161-181.

LAfli (E.) \& Meischner (J.)

2008 «Hellenistische und römische Grabstelen im Archäologischen Museum von Hatay in Antakya », Jahreshefte des Österreichischen Archäologischen Institutes in Wien 77, p. 145-183.

MAIURi (A.)

1925 Nuova silloge epigrafica di Rodi e Cos, Firenze.

Mitford (T. B.)

1961 «The Hellenistic inscriptions of Old Paphos », The Annual of the British School at Athens 56, p. 1-41.

Mooren (L.)

1977 La hiérarchie de cour ptolémaïque (Studia Hellenistica 23), Louvain. 
Peremans (W.), Van't Dack (E.), Mooren (L.) \& SWINNEN (W.)

1968

Prosopographia Ptolemaica, VI, La cour, les relations internationales et les possessions extérieures, la vie culturelle (Studia Hellenistica 17), Louvain.

RoBERT (L.)

1973 «Sur des inscriptions de Délos », Études déliennes ( $\mathrm{BCH}$, Supplément 1), Athènes, p. 435-466.

ROBERT (L.)

2007

Choix d'écrits, Paris.

SAulcy (L. de)

1853 Voyage autour de la mer Morte et dans les terres bibliques, exécuté de décembre 1850 à avril 1851, Paris.
Villa (N.), Rousseau (F.), Heilbrun (F.) \& Caubet (A.)

1982 F. de Saulcy (1807-1880) et la Terre Sainte, Paris.

WADDINGTON (W. H.)

1870

Inscriptions grecques et latines de la Syrie, Paris.

Yon (J.-B.)

2003 «À propos de la formule $\alpha \lambda^{2} v \pi \varepsilon \chi \alpha \tilde{\imath} \rho \varepsilon »$, Syria 80, p. 151-159.

Yon (J.-B.) \& Aliquot (J.)

2016

Inscriptions grecques et latines du Musée national de Beyrouth (Bulletin d'archéologie et d'architecture libanaises, Hors-Série 12), Beyrouth. 
